\title{
Newton-Euler, Lagrange and Kirchhoff formulations of rigid body dynamics: a unified approach
}

\author{
Enrico Massa · Stefano Vignolo
}

Received: date / Accepted: date

\begin{abstract}
A unified formulation of rigid body dynamics based on Gauss principle is proposed. The Lagrange, Kirchhoff and Newton-Euler equations are seen to arise from different choices of the quasi-coordinates in the velocity space. The group-theoretical aspects of the method are discussed.
\end{abstract}

Keywords Gauss principle · Rigid body kinematics and dynamics · Lagrange and Kirchhoff equations

PACS 45.40.-f · 45.20.D · 45.20.Jj

\section{Introduction}

Kirchhoff equations are a useful tool in rigid body dynamics: they are well known and widely used in many fields of applied mathematics, such as robotics, as well as aerospace and naval engineering.

For example, they play a central role in the representation of the hydrodynamical forces acting on a rigid body moving in an incompressible, irrotational, and inviscid fluid in terms of the so called added mass [1,2], an aspect that has significant applications in maneuvering models for surface vessels as well as for underwater marine vehicles [3-5].

The deduction of Kirchhoff equations from NewtonEuler ones is well known. On the contrary, the relationship between Kirchhoff and Lagrange equations is less

\section{E. Massa}

DIME, Sez. Metodi e Modelli Matematici, Università di Genova. Piazzale Kennedy, Pad. D. 16129 Genova (Italy).

E-mail:massa@dima.unige.it

S. Vignolo

DIME, Sez. Metodi e Modelli Matematici, Università di Genova. Piazzale Kennedy, Pad. D. 16129 Genova (Italy).

E-mail: vignolo@dime.unige.it immediate and, in the authors' knowledge, not readily available in the literature.

In this brief note we fill this gap at the light of Gauss principle of least constraint. The differences between the various formulations are shown to stem from different choices of the quasi coordinates in the velocity space. In particular, Kirchhoff and Newton-Euler approaches are seen to reflect the Lie group structure of the configuration manifold associated with the free rigid body, namely to involve quasi-coordinates respectively associated with left-invariant and right-invariant 1forms.

Besides pointing out the centrality of Gauss principle, the present contribution is aimed at illustrating how the invariance properties of the configuration manifolds help selecting families of independent variables especially suited to the formulation of the equations of motion. As such, it may provide a useful tool for possible applications in different engineering disciplines.

\section{The euclidean group}

In this section, a few general aspects of rigid body kinematics are briefly reviewed.

Given a rigid body $\mathfrak{B}$, let $\mathfrak{F}^{\prime}=\left\{O, \underline{e}_{1}, \underline{e}_{2}, \underline{e}_{3}\right\}$ be a body-fixed cartesian (positive) reference frame, with coordinates $x_{i}(P)=(P-O) \cdot \underline{e}_{i}=$ cost. $\forall P \in \mathfrak{B}$.

Denoting by $\mathfrak{F}=\left\{\Omega, \underline{k}_{1}, \underline{k}_{2}, \underline{k}_{3}\right\}$ a cartesian (positive) reference frame in the observer's space, with coordinates $\hat{x}_{i}(P)=(P-\Omega) \cdot \underline{k}_{i}$, every configuration of $\mathfrak{B}$ relative to $\mathfrak{F}$ is described by the affine transformation

$\hat{x}_{i}(P)=R_{i j} x_{j}(P)+b_{i}, \quad i, j=1,2,3$

with $R_{i j}:=\underline{k}_{i} \cdot \underline{e}_{j}$ and $b_{i}:=x_{i}(O)=(O-\Omega) \cdot \underline{k}_{i}$. 
The totality of transformations (1) form a Lie group $[6,7]$, henceforth denoted by $S E(3)$ and called the $e u$ clidean group.

Introducing the column vectors $\underset{\sim}{\hat{x}}={ }^{t}\left(\hat{x}_{1}, \hat{x}_{2}, \hat{x}_{3}, 1\right)$, $\underset{\sim}{\sim}{ }^{t}\left(x_{1}, x_{2}, x_{3}, 1\right)$, eq. (1) takes the compact form

$\hat{x}=\left(\begin{array}{cc}R_{i j} & b_{i} \\ 0 & 1\end{array}\right) \underset{\sim}{x}$

pointing out the isomorphism between $S E(3)$ and the subgroup of $G L(4)$ given by the semi-direct product of $S O(3)$ with $\mathbb{R}^{3}$.

In the given geometrical environment, every evolution of $\mathfrak{B}$ is described by a curve $\gamma: \mathbb{R} \rightarrow S E(3)$, namely

$\gamma(t)=\left(\begin{array}{cc}R_{i j}(t) & b_{i}(t) \\ 0 & 1\end{array}\right)$

Denoting by $\hat{u}_{i}=\underline{v}_{O} \cdot \underline{k}_{i}$ and $u_{i}=\underline{v}_{O} \cdot \underline{e}_{i}$ the components - respectively in the bases $\left\{\underline{k}_{i}\right\}$ and $\left\{\underline{e}_{i}\right\}$ - of the velocity $\underline{v}_{O}=\left.\frac{d}{d t}\right|_{\mathfrak{F}}(O-\Omega)$ of the body-fixed origin, we have the identifications

$\hat{u}_{i}=\dot{b}_{i}, \quad u_{i}=R_{j i} \dot{b}_{j} \quad \Longrightarrow \quad \hat{u}_{i}=R_{i j} u_{j}$

The angular velocity $\underline{\omega}$ of $\mathfrak{B}$ relative to the frame $\mathfrak{F}$ is similarly expressed in components in either form

$\underline{\omega}=\frac{1}{2} \underline{e}_{i} \wedge \underline{\dot{e}}_{i}=\frac{1}{2}\left(R^{t} \dot{R}\right)_{p q} \varepsilon_{p q r} \underline{k}_{r}:=\hat{\omega}_{r} \underline{k}_{r}$

$\underline{\omega}=\frac{1}{2} \underline{e}_{i} \wedge \underline{\dot{e}}_{i}=\frac{1}{2}\left({ }^{t} \dot{R} R\right)_{p q} \varepsilon_{p q r} \underline{e}_{r}:=\omega_{r} \underline{e}_{r}$

${ }^{t} \dot{R}$ and $\varepsilon_{p q r}$ indicating the transpose of the matrix $\dot{R}$ and the permutation symbol.

\section{Group-invariant velocities}

As pointed out, the euclidean group $S E(3)$ is the configuration space of the free rigid body. The tangent bundle $T S E(3)$ is therefore identical to the associated velocity space.

Every local coordinate system $q^{\alpha}(\alpha=1, \ldots, 6)$ in $S E(3)$ induces jet coordinates $q^{\alpha}, \dot{q}^{\alpha}$ in $T S E(3)$. The latter are the ones commonly adopted in Lagrangian Mechanics.

Other choices are of course available: for example, any 1-form $\sigma=\sigma_{\alpha} d q^{\alpha}$ on $S E(3)$ determines a corresponding function $\sigma_{\alpha} \dot{q}^{\alpha}$ that, under suitable circumstances, can be adopted as fiber coordinate in TSE(3).

In this respect, the left and right invariant 1-forms are naturally highlighted as distinguished geometrical objects, intimately related to the symmetries of the underlying environment.
Any function on $T S E(3)$ associated with a (left or right) invariant 1 -form $\sigma \in \mathcal{D}_{1}(S E(3))$ will be called a group-invariant velocity.

A left-invariant basis for the module $\mathcal{D}_{1}(S E(3))$ is given by

$\mu_{i}=R_{j i} d b_{j}$

$\nu_{i}=-\frac{1}{2} \varepsilon_{i p q}\left({ }^{t} R d R\right)_{p q}=-\frac{1}{2} \varepsilon_{i p q} R_{k p} d R_{k q}$

Denoting by $l_{g}: S E(3) \rightarrow S E(3)$ the left transport $l_{g}(h):=g \cdot h$, we have in fact the relation

$l_{g}^{*}\left(\begin{array}{cc}R_{i j} & b_{i} \\ 0 & 1\end{array}\right)=\left(\begin{array}{cc}R_{i p}(g) R_{p j} & R_{i j}(g) b_{j}+b_{i}(g) \\ 0 & 1\end{array}\right)$

whence, by straightforward calculations

$l_{g}^{*}\left(\mu_{i}\right)=R_{j p}(g) R_{p i} R_{j k}(g) d b_{k}=\mu_{i}$

$l_{g}^{*}\left(\nu_{i}\right)=-\frac{1}{2} \varepsilon_{i p q} R_{k s}(g) R_{s p} R_{k l}(g) d R_{l q}=\nu_{i}$.

The generalized velocities associated with the 1forms (6) are

$\begin{aligned} \mu_{i} & \mapsto \quad R_{j i} \dot{b}_{j}=\underline{v}_{O} \cdot \underline{e}_{i}=u_{i} \\ \nu_{i} & \mapsto \quad-\frac{1}{2} \varepsilon_{i p q} R_{k p} \dot{R}_{k q}=\omega \cdot \underline{e}_{i}=\omega_{i}\end{aligned}$

i.e. they coincide with the components, in the bodyfixed basis, of the vectors $\underline{v}_{O}$ and $\underline{\omega}$ involved in the representation

$\underline{v}_{P}=\underline{v}_{O}+\underline{\omega}_{\wedge}(P-O), \quad P \in \mathfrak{B}$

A similar analysis shows that the 1 -forms

$\hat{\mu}_{i}=d b_{i}+R_{i p} d R_{j p} b_{j}$

$\hat{\nu}_{i}=\frac{1}{2} \varepsilon_{i p q}\left(R d^{t} R\right)_{p q}=\frac{1}{2} \varepsilon_{i p q} R_{p k} d R_{q k}$

form a right-invariant basis for the module $\mathcal{D}_{1}(S E(3))$.

Denoting by $r_{g}(h):=h \cdot g$ the right transport and arguing as above, we have in fact the relations

$r_{g}^{*}\left(R_{i j}\right)=R_{i p} R_{p j}(g), \quad r_{g}^{*}\left(b_{i}\right)=R_{i j} b_{j}(g)+b_{i}$

whence

$r_{g}^{*}\left(\hat{\mu}_{i}\right)=d b_{i}+R_{i p} d R_{j p} b_{j}=\hat{\mu}_{i}$

$r_{g}^{*}\left(\hat{\nu}_{i}\right)=\frac{1}{2} \varepsilon_{i p q} R_{p s} R_{s k}(g) d R_{q l} R_{l k}(g)=\hat{\nu}_{i}$.

The generalized velocities associated with the 1forms (9) are

$$
\begin{gathered}
\hat{\mu}_{i} \mapsto \quad \dot{b}_{i}+R_{i p} \dot{R}_{j p} b_{j}=\dot{b}_{i}+\varepsilon_{i j r} b_{j} \hat{\omega}_{p}= \\
=\left(\underline{v}_{O}+\underline{\omega} \wedge(\Omega-O)\right) \cdot \underline{k}_{i}:=\hat{\xi}_{i} \\
\hat{\nu}_{i} \mapsto \quad \mapsto \quad \frac{1}{2} \varepsilon_{i p q} R_{k p} \dot{R}_{k q}=\hat{\omega}_{i}
\end{gathered}
$$


i.e. they coincide with the components, in the observer's frame, of the vectors $\underline{\xi}$ and $\underline{\omega}$ involved in the less usual representation ${ }^{1}$

$\underline{v}_{P}=\underline{\xi}+\underline{\omega} \wedge(P-\Omega)$

\section{The equations of motion}

A central point in the development of mechanics in the presence of constraints is the characterization of the reactive forces. A milestone in this sense is provided by the following

Gauss Principle. For a material system subject to ideal constraints, the actual motion under the action of given active forces is selected among the totality of kinematically admissible evolutions by the requirement that, in any kinetic state $\left(t, P_{1}, \ldots, P_{N}, \underline{v}_{1}, \ldots, \underline{v}_{N}\right)$, the accelerations $\underline{a}_{1}, \ldots, \underline{a}_{N}$ are those for which the function

$C:=\frac{1}{2} \sum_{i=1}^{N} m_{i}\left|\underline{a}_{i}-\frac{\underline{F}_{i}}{m_{i}}\right|^{2}$

attains a minimum.

For holonomic systems, Gauss' Principle is equivalent to d'Alembert's principle of virtual work [8-12]. The advantages of Gauss' formulation are its applicability to a wider class of constraints, including the kinetic ones [11], and its adaptedness to the language of quasi-coordinates.

The implementation of the algorithm is straightforward: in terms of generic (fibred) coordinates $t, q^{\alpha}, z^{\alpha}$ on the velocity space, we have the relations

$\dot{q}^{\alpha}=\psi^{\alpha}\left(t, q^{1}, \ldots, q^{n}, z^{1}, \ldots, z^{n}\right)$

essentially equivalent to a definition of the generalized velocities, as well as the representations

$P_{i}=P_{i}\left(t, q^{1}, \ldots, q^{n}\right)$

$\underline{v}_{i}=\frac{\partial P_{i}}{\partial t}+\frac{\partial P_{i}}{\partial q^{\alpha}} \psi^{\alpha}=\underline{v}_{i}\left(t, q^{\alpha}, z^{\alpha}\right)$

1 According to eq. (10a), the vector $\xi$ represents the velocity of the point of the body $\mathfrak{B}$ instantly located at the space origin of the observer's frame. As such, it may look a rather factitious object. A better understanding of the symmetry hidden in the representation (11) is gained interpreting the vectors $-\underline{\xi},-\underline{\omega}$ respectively as the linear and angular velocity of the frame $\mathfrak{F}$ relative to $\mathfrak{F}^{\prime}$, and the vector $-\underline{v}_{P}$ as the velocity, relative to $\mathfrak{F}^{\prime}$, of a point $P$ at rest in $\mathfrak{F}$. In this way, eq. (11) is on the same footing as eq. (8), namely it describes, up to a sign, the rigid motion of $\mathfrak{F}$ relative to $\mathfrak{F}^{\prime}$. Interchanging left and right invariance is therefore equivalent to interchanging the roles of the frames $\mathfrak{F}$ and $\mathfrak{F}^{\prime}$, i.e. to replacing each transformation by the corresponding inverse.
At each kinetic state, the expression of the admissible accelerations takes therefore the form

$\underline{a}_{i}=\frac{\partial \underline{v}_{i}}{\partial t}+\frac{\partial \underline{v}_{i}}{\partial q^{\alpha}} \psi^{\alpha}+\frac{\partial \underline{v}_{i}}{\partial z^{\alpha}} \dot{z}^{\alpha}$

involving $n$ additional variables $\dot{z}^{\alpha}$, interpretable as coordinates along the fibres an affine bundle over the velocity space, known as the second tangent bundle [13].

It is then clear that imposing Gauss principle means, for each choice of $t, q^{\alpha}, z^{\alpha}$, minimizing the function (12) with respect to the variables $\dot{z}^{1}, \ldots, \dot{z}^{n}$. On account of the identity $\frac{\partial \underline{a}_{i}}{\partial \dot{z}^{\alpha}}=\frac{\partial \underline{v}_{i}}{\partial z^{\alpha}}$, this entails the condition

$\frac{\partial C}{\partial \dot{z}^{\alpha}}=\sum_{i=1}^{N}\left(m_{i} \underline{a}_{i}-\underline{F}_{i}\right) \cdot \frac{\partial \underline{v}_{i}}{\partial z^{\alpha}}=0$

more conveniently written as

$\frac{d}{d t}\left(\frac{\partial T}{\partial z^{\alpha}}\right)-\sum_{i=1}^{N} m_{i} \underline{v}_{i} \frac{d}{d t}\left(\frac{\partial \underline{v}_{i}}{\partial z^{\alpha}}\right)=\sum_{i=1}^{N} \underline{F}_{i} \cdot \frac{\partial \underline{v}_{i}}{\partial z^{\alpha}}$

Eqs. (14) may be viewed as a set of equations for the determination of the unknowns $\dot{z}^{\alpha}$ in terms of the kinetic variables $t, q^{\alpha}, z^{\alpha}{ }^{2}$. We let the reader verify that the positive-definiteness of the matrix $\frac{\partial^{2} C}{\partial \dot{z}^{\alpha} \partial \dot{z}^{\beta}}=$ $=\sum_{i} m_{i} \frac{\partial \underline{v}_{i}}{\partial z^{\alpha}} \cdot \frac{\partial \underline{v}_{i}}{\partial z^{\beta}}$ ensures both the solvability of the equations and the fact that they do indeed determine a minimum of the function $C$.

Summing up, we conclude that, eqs. (14), completed with the kinematical relations (13), determine the evolution of the system from given initial data through a well-posed Cauchy problem.

The covariance of the algorithm ensures that different choices of the generalized velocities lead to different but equivalent representations of the system (13), (14), without affecting the essence of the problem of motion, namely the determination of the curve $q^{\alpha}=q^{\alpha}(t)$ in configuration space.

As implicit in the notation, all previous results apply to discrete systems. In the continuous scheme, more suited to rigid body mechanics, the conclusions are essentially the same, with the concentrated attributes $m_{i}$, $\underline{F}_{i}$ replaced by corresponding measures $d m, d \underline{F}$ over the abstract space $\mathfrak{B}$ formed by the totality of points

2 Although conceptually preferable, for holonomic systems Gauss' principle is not strictly necessary in order to establish eq. (14): one may equally well start with d'Alembert's principle, make use of the identity $\frac{\partial P_{i}}{\partial q^{\alpha}}=\frac{\partial \underline{v}_{i}}{\partial \dot{q}^{\alpha}}$, and replace the resulting equations by the linear combinations

$0=\sum_{i=1}^{N}\left(m_{i} \underline{a}_{i}-\underline{F}_{i}\right) \cdot \frac{\partial \underline{v}_{i}}{\partial \dot{q}^{\beta}} \frac{\partial \dot{q}^{\beta}}{\partial z^{\alpha}}=\sum_{i=1}^{N}\left(m_{i} \underline{a}_{i}-\underline{F}_{i}\right) \cdot \frac{\partial \underline{v}_{i}}{\partial z^{\alpha}}$ 
of the body, and with eqs. (14) replaced by the integral relations

$\frac{d}{d t}\left(\frac{\partial T}{\partial z^{\alpha}}\right)-\int_{\mathfrak{B}} \underline{v}_{P} \frac{d}{d t}\left(\frac{\partial \underline{v}_{P}}{\partial z^{\alpha}}\right) d m=\int_{\mathfrak{B}} \frac{\partial \underline{v}_{P}}{\partial z^{\alpha}} \cdot d \underline{F}$

Coming to the problem in study, let us now verify that, depending on the choice of the generalized velocities, eqs. (15) yield back the Lagrange, Kirchhoff and Newton-Euler equations of motion.

- Jet coordinates: with the ansatz $z^{\alpha}=\dot{q}^{\alpha}$, eqs. (15) entail the Lagrange equations

$\frac{d}{d t}\left(\frac{\partial T}{\partial \dot{q}^{\alpha}}\right)-\frac{\partial T}{\partial q^{\alpha}}=\int_{\mathfrak{B}} \frac{\partial \underline{v}_{P}}{\partial \dot{q}^{\alpha}} \cdot d \underline{F}=\int_{\mathfrak{B}} \frac{\partial P}{\partial q^{\alpha}} \cdot d \underline{F}$

the right-hand sides expressing the so-called generalized forces. The argument is well known, and does not require any comment.

- Left-invariant velocities: as shown by eq. (7), they correspond to the choice $z^{i}=u_{i}, z^{3+i}=\omega_{i}, i=1,2,3$, $u_{i}=\underline{v}_{O} \cdot \underline{e}_{i}$ and $\omega_{i}=\underline{\omega} \cdot \underline{e}_{i}$ expressing the components of the linear and angular velocity of $\mathfrak{B}$ in the bodyfixed frame. On account of eq. (8), this entails the identities

$\frac{\partial \underline{v}_{P}}{\partial u_{k}}=\underline{e}_{k}, \quad \frac{\partial \underline{v}_{P}}{\partial \omega_{k}}=\underline{e}_{k} \wedge(P-O)$

From eqs. (16), respectively denoting by $\underline{Q}, \underline{\Gamma}_{0}, \underline{R}$ and $\underline{M}_{O}$ the total linear momentum, the angular momentum (with respect to $O$ ), the resultant of the external forces and the external torque relative to $O$, we have the identifications

$\frac{\partial T}{\partial u_{k}}=\int_{\mathfrak{B}} \underline{v}_{P} d m \cdot \underline{e}_{k}=\underline{Q} \cdot \underline{e}_{k}:=Q_{k}$

$\frac{\partial T}{\partial \omega_{k}}=\int_{\mathfrak{B}} \underline{v}_{P} \cdot \underline{e}_{k} \wedge(P-O) d m=\underline{\Gamma}_{0} \cdot \underline{e}_{k}:=\Gamma_{k}$

$\int_{\mathfrak{B}} \frac{\partial \underline{v}_{P}}{\partial u_{k}} \cdot d \underline{F}=\underline{R} \cdot \underline{e}_{k}:=R_{k}$

$\int_{\mathfrak{B}} \frac{\partial \underline{v}_{P}}{\partial \omega_{k}} \cdot d \underline{F}=\underline{M}_{O} \cdot \underline{e}_{k}:=M_{k}$

Inserting eqs. (16), (17) into (15) and recalling the Poisson formulae, we end up with the equations

$\frac{d}{d t}\left(\frac{\partial T}{\partial u_{k}}\right)+\varepsilon_{k p q} \omega_{p} \frac{\partial T}{\partial u_{q}}=R_{k}$

$\frac{d}{d t}\left(\frac{\partial T}{\partial \omega_{k}}\right)+\varepsilon_{k p q}\left(\omega_{p} \frac{\partial T}{\partial \omega_{q}}+u_{p} \frac{\partial T}{\partial u_{q}}\right)=M_{k}$

identical to the Kirchhoff equations.

- Right-invariant velocities: according to eq. (10), they correspond to the ansatz $z^{i}=\hat{\xi}_{i}, z^{3+i}=\hat{\omega}_{i}, i=1,2,3$, $\hat{\xi}_{i}=\underline{\xi} \cdot \underline{k}_{i}$ and $\hat{\omega}_{i}=\underline{\omega} \cdot \underline{k}_{i}$ being the components in the observer's frame of the vectors involved in the representation We have therefore the identifications

$\frac{\partial \underline{v}_{P}}{\partial \hat{\xi}_{j}}=\underline{k}_{j}, \quad \frac{\partial \underline{v}_{P}}{\partial \hat{\omega}_{j}}=\underline{k}_{j} \wedge(P-\Omega)$

From these, preserving the notation $\underline{Q}, \underline{R}$ for the total momentum and total external force, and denoting by $\underline{\Gamma}_{\Omega}, \underline{M}_{\Omega}$ respectively the angular momentum and the torque with respect to the fixed origin $\Omega$, we get the relations

$\frac{\partial T}{\partial \hat{\xi}_{s}}=\underline{Q} \cdot \underline{k}_{s}:=\hat{Q}_{s}, \quad \frac{\partial T}{\partial \hat{\omega}_{s}}=\underline{\Gamma}_{\Omega} \cdot \underline{k}_{s}:=\hat{\Gamma}_{s}$

$\int_{\mathfrak{B}} \frac{\partial \underline{v}_{i}}{\partial \hat{\xi}_{s}} \cdot d \underline{F}=\underline{R} \cdot \underline{k}_{s}:=\hat{R}_{s}$

$\int_{\mathfrak{B}} \frac{\partial \underline{v}_{i}}{\partial \hat{\omega}_{s}} \cdot d \underline{F}=\underline{M}_{\Omega} \cdot \underline{k}_{s}:=\hat{M}_{s}$

As above, inserting eqs. (18), (19) into (15) yields the equations

$\frac{d \hat{Q}_{s}}{d t}=\hat{R}_{s}$

$\frac{d \hat{\Gamma}_{s}}{d t}=\hat{M}_{s}$

identical to the Newton-Euler equations.

- "Hybrid" formulation: for completeness, we point out a further representation of the equations of motion, based on the choice $z^{i}=\dot{b}_{i}, z^{3+i}=\omega_{i}, i=1,2,3$, i.e. involving the components of the velocity $\underline{v}_{O}$ in the observer's frame and the components of the angular velocity $\underline{\omega}$ in the body-fixed frame.

Referring to eq. (8), we have now the identities

$\frac{\partial \underline{v}_{P}}{\partial \dot{b}_{s}}=\underline{k}_{s}, \quad \frac{\partial \underline{v}_{P}}{\partial \omega_{s}}=\underline{e}_{s} \wedge(P-O)$

whence, keeping the same notation as above

$\frac{\partial T}{\partial \dot{b}_{s}}=\hat{Q}_{s}, \quad \frac{\partial T}{\partial \omega_{s}}=\Gamma_{s}$

$\int_{\mathfrak{B}} \frac{\partial \underline{v}_{P}}{\partial \dot{b}_{s}} \cdot d \underline{F}=\hat{R}_{s}, \quad \int_{\mathfrak{B}} \frac{\partial \underline{v}_{P}}{\partial \omega_{s}} \cdot d \underline{F}=M_{s}$

Substituting into eqs. (15) yields the required equations, synthetically expressed in vector form as

$\frac{d}{d t} \underline{Q}=\underline{R}$

$\left.\frac{d}{d t}\right|_{\mathfrak{F}^{\prime}} \underline{\Gamma}_{O}+\underline{\omega} \wedge \underline{\Gamma}_{O}+\underline{v}_{O} \wedge \underline{Q}=\underline{M}_{O}$

These reproduce once again the content of the NewtonEuler equations, with the angular momentum and the torque now referred to the body-fixed point $O$. 


\section{References}

1. L. M. Milne-Thomson L.M., Theoretical Hydrodynamics, Dover Publications Inc., New York, USA (1968).

2. J. N. Newman, Marine Hydrodynamics, The MIT Press, Cambridge, USA (1977).

3. T. I. Fossen, Marine Control Systems, Marine Cybernetics, Trondheim, Norway (2002).

4. T. I. Fossen, Handbook of Marine Craft Hydrodynamics and Motion Control, John Wiley and Sons Ltd, Chichester, U.K. (2011).

5. E. M. Lewandowski, The Dynamics of Marine Craft, World Scientific Publishing Co., Singapore (2004).

6. F. W. Warner, Foundations of Differentiable Manifolds and Lie Groups, Springer-Verlag, New York, USA (1983).

7. S. Sternberg, Group Theory and Physics, Cambridge University Press, Cambridge (1994).

8. T. Levi-Civita and U. Amaldi, Lezioni di Meccanica Razionale, Zanichelli, Bologna (1984).

9. E.T. Whittaker, A Treatise on the Analytical Dynamics of Particles and Rigid Bodies, (4th ed.), Cambridge University Press, Cambridge (1959).

10. G. Hamel, Theoretische Mechanik. Eine einheitliche Einfuhrung in die gesamte Mechanik, Springer-Verlag, Berlin (1949).

11. E. Massa and E. Pagani, Classical Dynamics of nonholonomic systems: a geometric approach, Ann. Inst. Henri Poincaré, Physique théorique 55, 511-544 (1991).

12. E. Massa and E. Pagani, A new look at Classical Mechanics of constrained systems, Ann. Inst. Henri Poincaré, Physique théorique 66, 1-36 (1997).

13. C. Godbillon, Geometrie differentielle et mecanique analytique, Hermann, Paris (1969). 